Supporting information

\title{
Phase Engineered Molybdenum Telluride/Black Phosphorus Van der Waals Heterojunctions for Tunable Multivalued Logic
}

\author{
Yasir Hassan ${ }^{1, \dagger}$, Pawan Kumar Srivastava ${ }^{2, \dagger}$, Budhi Singh ${ }^{3}$, Muhammad Sabbtain Abbas ${ }^{4}$, Fida \\ Ali $^{1}$, Won Jong Yoo ${ }^{1}$, Changgu Lee ${ }^{1,2, *}$ \\ ${ }^{1}$ SKKU Advanced Institute of Nanotechnology (SAINT), Sungkyunkwan University, Suwon \\ 16419, South Korea \\ ${ }^{2}$ School of Mechanical Engineering, Sungkyunkwan University, Suwon 16419, South Korea \\ ${ }^{3}$ Inter University Accelerator Centre, Aruna Asaf Ali Marg, New Delhi 110067, India \\ ${ }^{4}$ Department of Physics, Sungkyunkwan University, Suwon, 16419, South Korea \\ ${ }^{\dagger}$ These authors contributed equally to work. \\ *Email: peterlee@skku.edu
}

$\underline{\text { Index. }}$

S1. Current-voltage characteristics of BP/MoTe $2\left(1 \mathrm{~T}^{\prime}+2 \mathrm{H}\right)$ : Evidence of type-I heterojunction

$\mathrm{S} 2$. High resolution transmission electron image of $\mathrm{BP} / \mathrm{MoTe}_{2}$ heterojunction interface.

S3. Energy dispersive X-ray spectroscopy of $\mathrm{MoTe}_{2}$ for different thickness.

S4. Activation energy at different $\mathrm{V}_{\mathrm{D}}$ for $2 \mathrm{H}-\mathrm{MoTe}_{2}$ and $2 \mathrm{H}+1 \mathrm{~T}^{\prime} \mathrm{MoTe}_{2}$.

S5. Output characteristics of (a) $21 \mathrm{~nm}$ thick BP and (b) $2 \mathrm{H}+1 \mathrm{~T}^{\prime} \mathrm{MoTe}_{2}(16 \mathrm{~nm})$

S6. $\mathrm{V}_{\mathrm{D}}$ dependent band alignment at $\mathrm{BP} / 2 \mathrm{H}-\mathrm{MoTe}_{2}$ heterojunction 
S1. Current-voltage characteristics of $\mathrm{BP} / \mathrm{MoTe}_{2}\left(1 \mathrm{~T}^{\prime}+2 \mathrm{H}\right)$ : Evidence of type-I heterojunction
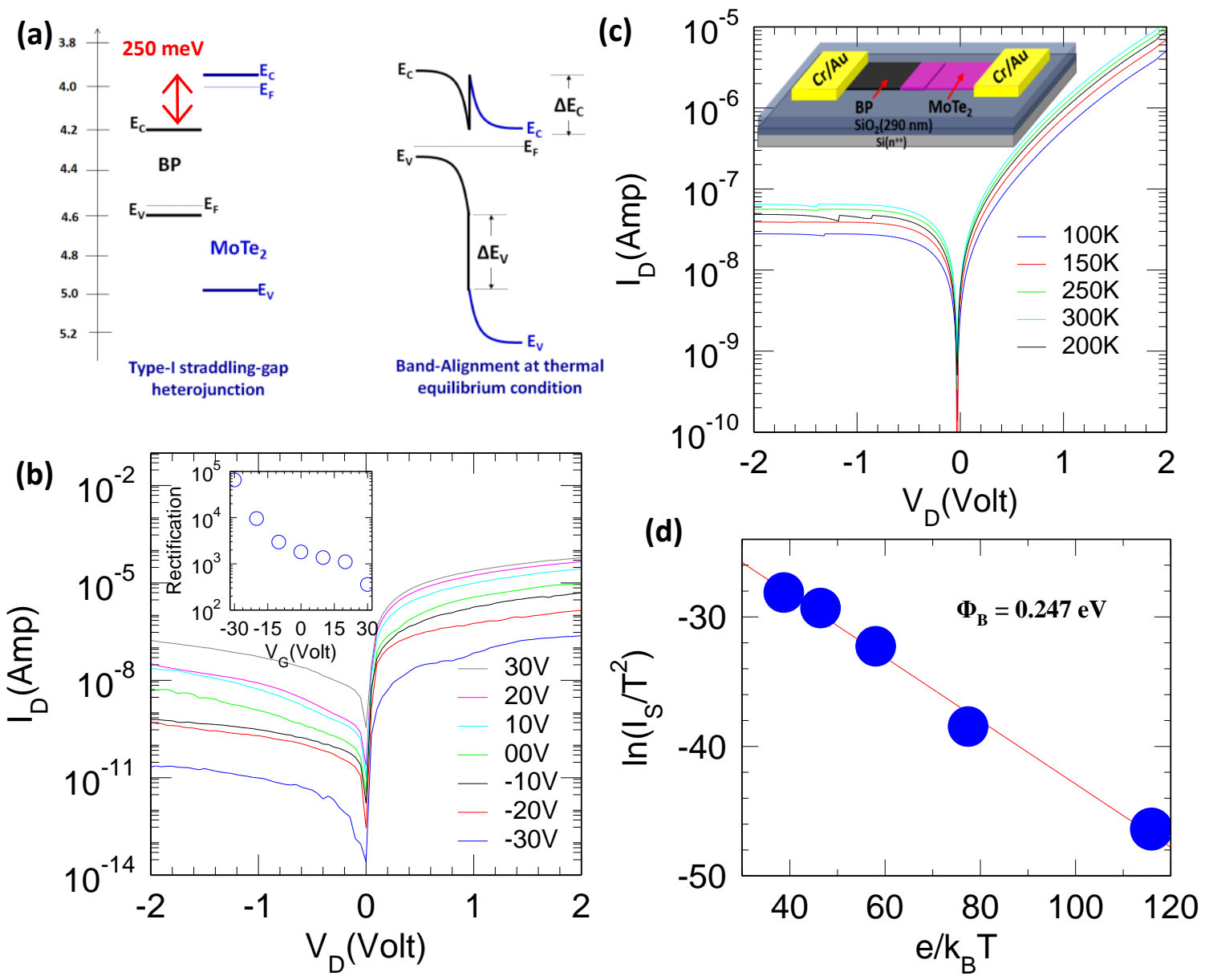
Figure S1. (a) Band alignment at BP $(16 \mathrm{~nm}) / \mathrm{MoTe}_{2}(8 \mathrm{~nm})$ heterojunction. (b) Gate dependent current-voltage characteristics of $\mathrm{BP} / \mathrm{MoTe}_{2} \mathrm{p}$-n heterojunction. Inset shows gate tunable current-rectification. (c) Temperature dependent current-transport in $\mathrm{BP} / \mathrm{MoTe}_{2} \mathrm{p}$-n heterojunction. Inset shows schematics of the device. Heterojunction overlapped region is around $5 \mu \mathrm{m}^{2}$ and channel length is $8 \mu \mathrm{m}$. (d) Energy barrier height $\left(\Phi_{\mathrm{B}}\right)$ extraction from $\ln \left(I_{S} / T^{2}\right)$ vs $1 / T$ graph ${ }^{1} . \Phi_{\mathrm{B}}=0.247 \mathrm{eV}$ is very close to reported values of conduction band offset between BP and $\mathrm{MoTe}_{2}\left(\right.$ refs. $\left.^{2-4}\right)$. It reveals the type-I behavior of BP/MoTe $2 p-n$ heterojunction.

\section{S2. High resolution transmission electron image of $\mathrm{BP} / \mathrm{MoTe}$ heterojunction interface.}

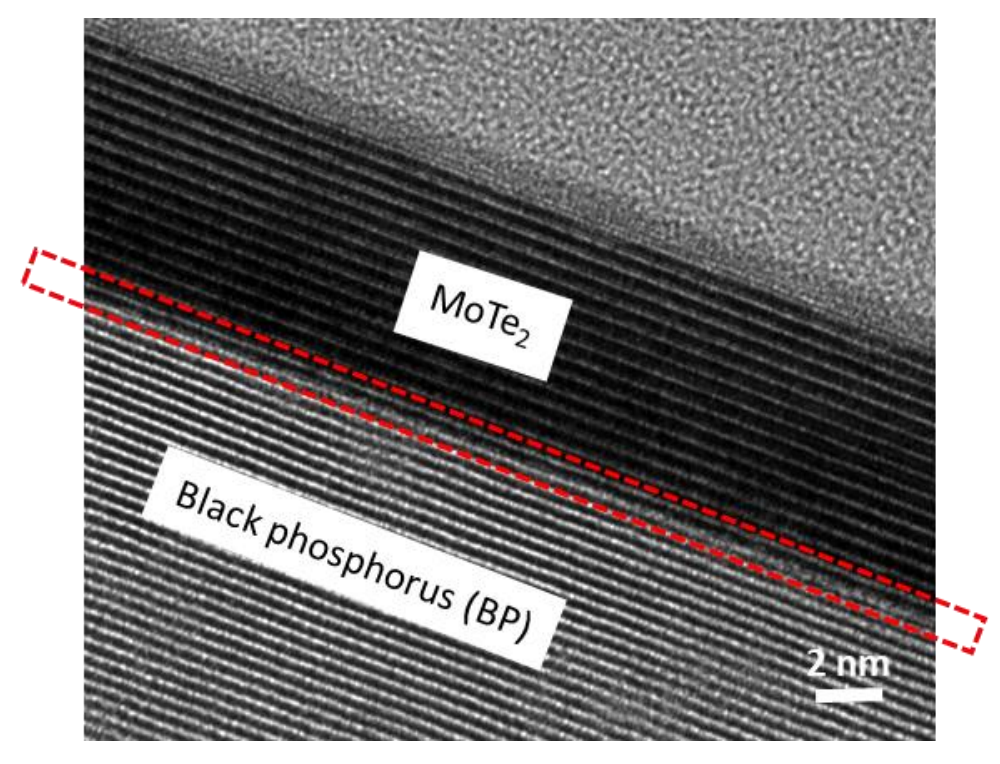

Figure S2. High resolution transmission electron microscope image of BP/MoTe 2 interface of one of the measured device. An ultraclean $\mathrm{BP} / \mathrm{MoTe}_{2}$ interface at can be seen. $\mathrm{BP} / \mathrm{MoTe}_{2}$ heterostructures were fabricated on $\mathrm{SiO}_{2} / \mathrm{Si}$ substrates using standard mechanical transfer procedure. Then focused ion beam milling was used to cut off a small portion of the heterojunction and then transferred to TEM grid for cross sectional HRTEM/STEM imaging. Acceleration voltage for TEM measurement was $200 \mathrm{keV}$. 


\section{S3: Energy dispersive X-ray spectroscopy of MoTe2 for different thickness}

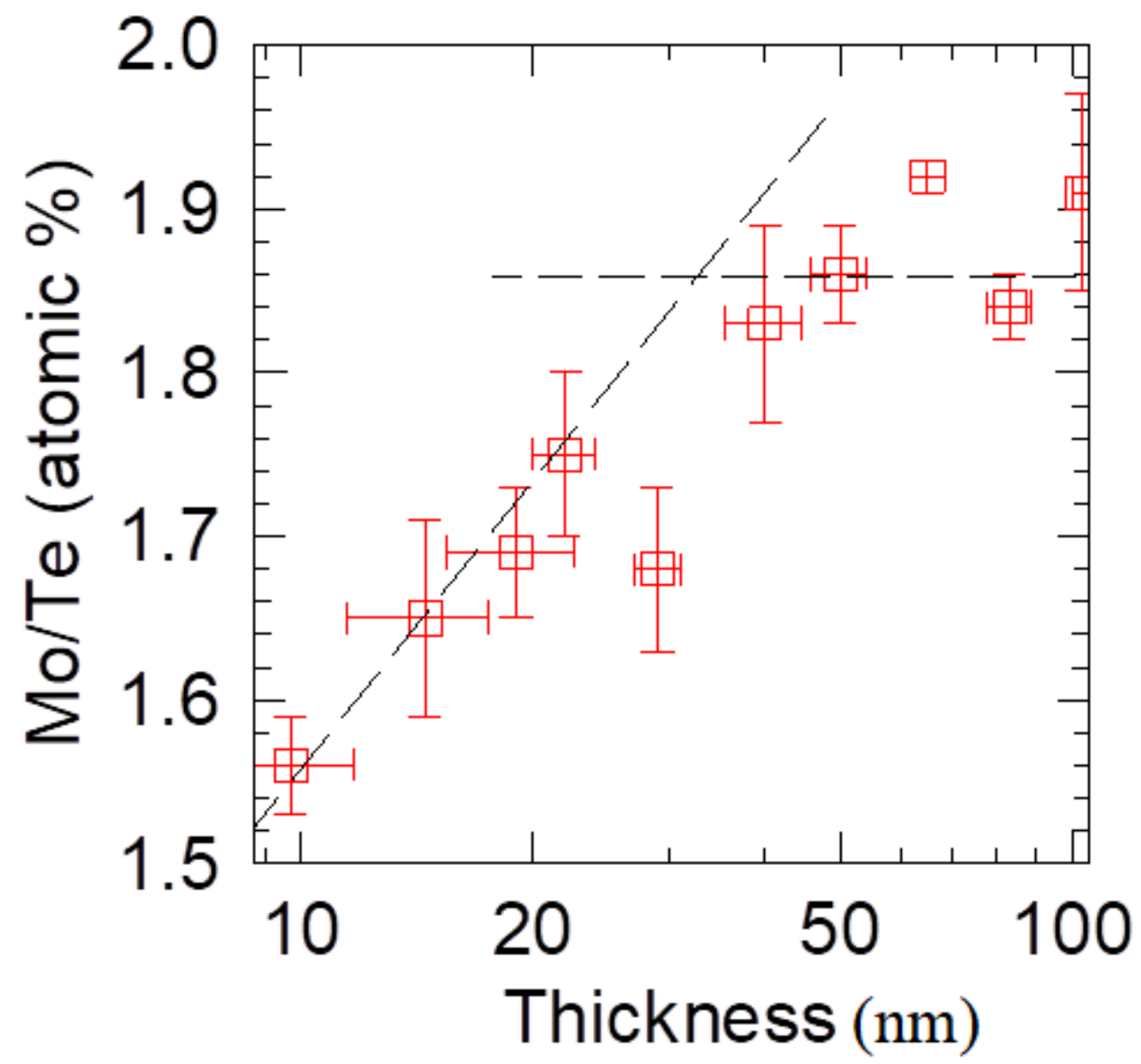

Figure S3. Semi-logarithmic plot of Molybdenum (Mo) and Tellurium (Te) atomic percentage ratio vs thickness of $\mathrm{MoTe}_{2}$ samples. Mo and Te atomic percentage in various $\mathrm{MoTe}_{2}$ flakes having different thickness was measured using energy dispersive X-ray spectroscopy in a scanning electron microscope. 


\section{S4: Activation energy at different $V_{D}$ for $2 \mathrm{H}-\mathrm{MoTe} 2$ and $2 \mathrm{H}+1 \mathrm{~T}^{\prime} M o \mathrm{Te}_{2}$}

(a)

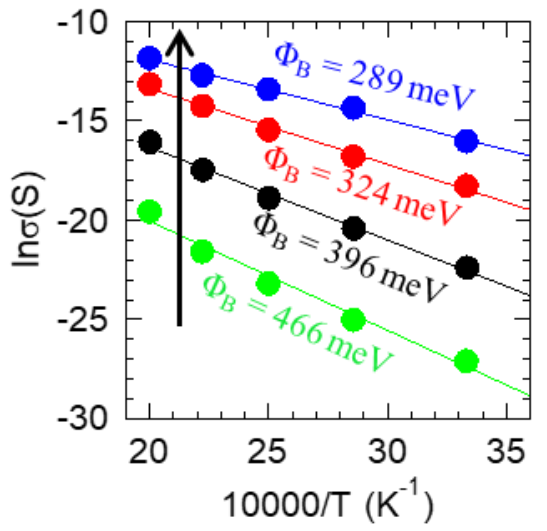

(c)

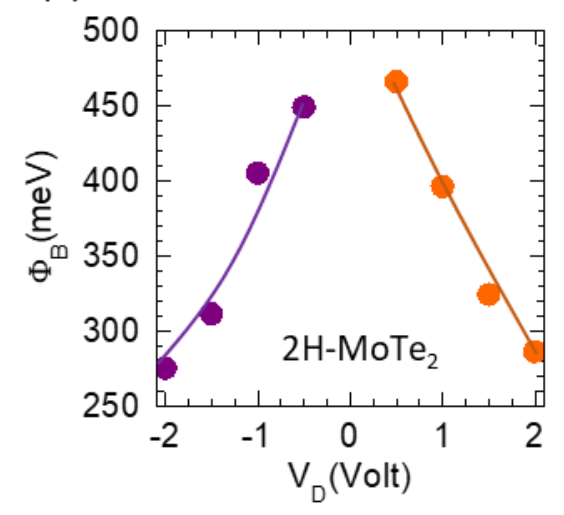

(b)

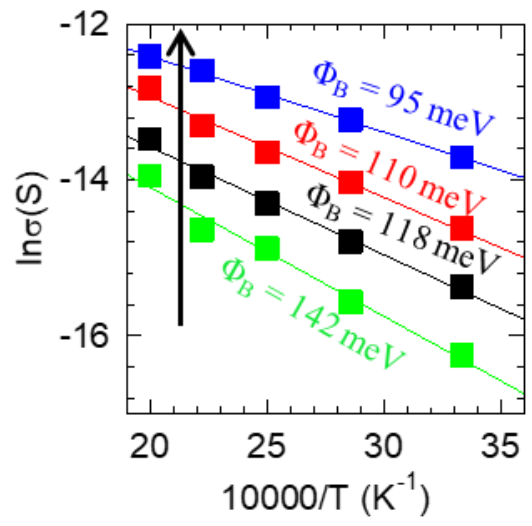

(d)

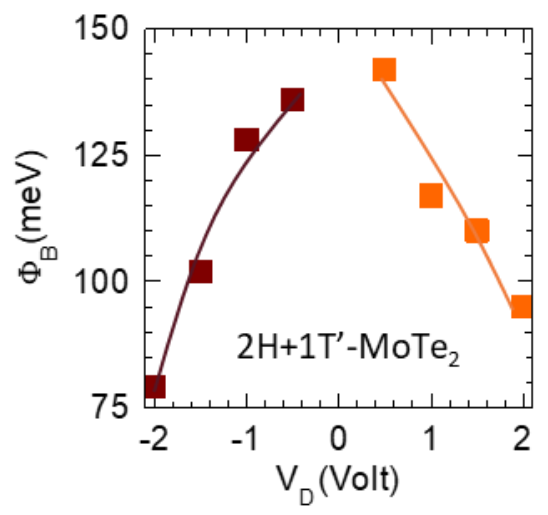

Figure S4. Arrhenius plot of conductance for (a) $2 \mathrm{H}-\mathrm{MoTe}_{2}$ and (b) $2 \mathrm{H}+1 \mathrm{~T}{ }^{\prime} \mathrm{MoTe}_{2} \mathrm{FETs}$ at various $\mathrm{V}_{\mathrm{D}}$ values ranging between $0.5 \mathrm{~V}$ to $2 \mathrm{~V}$ (in the direction of arrows). Magnitude of energy barriers are labelled in the graph and also summarized in Figure S4-c and S4-d. Symbols are the data points and connecting lines are guide to eyes. 


\section{S5: Output characteristics of (a) $21 \mathrm{~nm}$ thick BP and (b) $2 \mathrm{H}+1 \mathrm{~T}^{\prime} \mathrm{MoTe}_{2}(16 \mathrm{~nm})$}
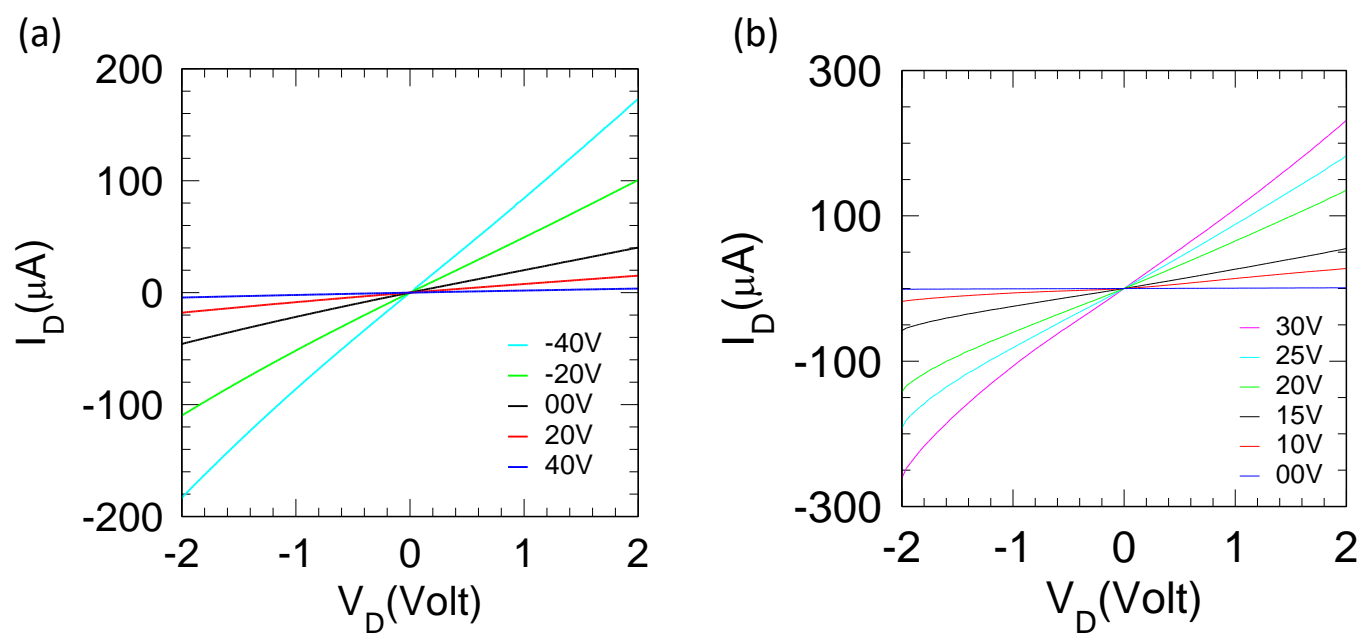

Figure S5. Output characteristics of (a) $21 \mathrm{~nm}$ thick BP and (b) $2 \mathrm{H}+1 \mathrm{~T}^{\prime} \mathrm{MoTe}_{2}(16 \mathrm{~nm})$. Current-transport in BP and $2 \mathrm{H}+1 \mathrm{~T}^{\prime} \mathrm{MoTe}_{2}$ FETs exhibit Ohmic behavior. 


\section{S6: Drain voltage $\left(V_{D}\right)$ dependent band alignment at $\mathrm{BP} / 2 \mathrm{H}-\mathrm{MoTe}_{2}$ heterojunction}

Energy band alignment at the $\mathrm{BP} / 2 \mathrm{H}-\mathrm{MoTe}_{2} \mathrm{vdW}$ heterojunction under different $\mathrm{V}_{\mathrm{D}}$ is illustrated in Figure S6. At $\mathrm{V}_{\mathrm{D}}>0 \mathrm{~V}$, the valance band (VB) of the BP side will bend downwards due to shift of Fermi level. At small $V_{D}$, the barrier for hole is large. However there is no barrier for electrons. Further increase in $V_{D}$ will cause bending of the VB of BP beyond the VB of $\mathrm{MoTe}_{2}$. At $\mathrm{V}_{\mathrm{D}} \approx 1 \mathrm{~V}$, the barrier for holes diminishes completely and the current-transport is dominated by the $\mathrm{BP}$ channel. Therefore, $\mathrm{V}_{\mathrm{G}}$ controls the channel more effectively at larger $\mathrm{V}_{\mathrm{D}}$ where no barrier exists to restrict the current-transport. This is the reason why we have observed larger peak to-valley current ratio and faster decrease in current with increasing $V_{D}$. At large $V_{D}$, change in the threshold voltage of the BP FET and lowering of the barrier for holes lead to a larger parallel region between $\mathrm{BP} / 2 \mathrm{H}-\mathrm{MoTe}_{2} \mathrm{vdW}$ heterojunction and the BP FET. Hence, the formation of this unique window is responsible for ternary logic inverter.

\section{Vacuum Level}

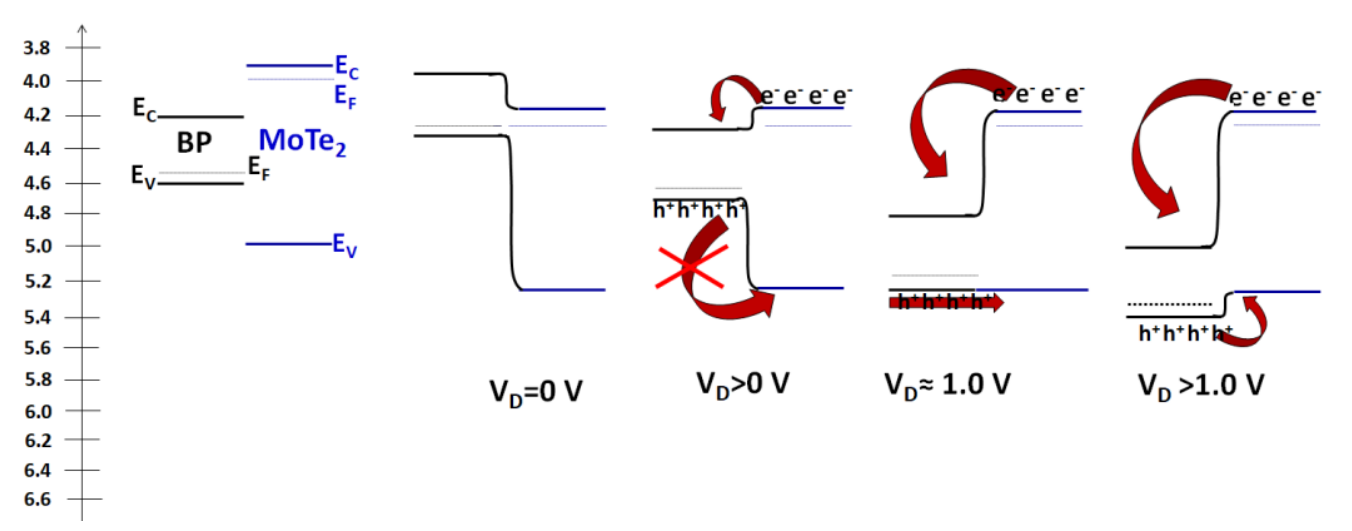

Figure S6. $\mathrm{V}_{\mathrm{D}}$ dependent band alignment at $\mathrm{BP} / 2 \mathrm{H}-\mathrm{MoTe}_{2}$ heterojunction. Conduction band minimum and valence band maximum values for $\mathrm{BP}$ and $\mathrm{Mote}_{2}$ have been adapted from references 2-4. 


\section{Supplementary references.}

(1) Keum, D. H.; Cho, S.; Kim, J. H.; Choe, D. H.; Sung, H. J.; Kan, M.; Kang, H.; Hwang, J. Y.; Kim, S. W.; Yang, H.; et al. Bandgap Opening in Few-Layered Monoclinic MoTe 2. Nat. Phys. 2015, 11 (6), 482-486.

(2) Zhang, C.; Gong, C.; Nie, Y.; Min, K. A.; Liang, C.; Oh, Y. J.; Zhang, H.; Wang, W.; Hong, S.; Colombo, L.; Wallace, R. M.; Cho, K. Systematic Study of Electronic Structure and Band Alignment of Monolayer Transition Metal Dichalcogenides in Van Der Waals Heterostructures. 2D Mater. 2017, 4, 015026.

(3) Huang, H. H.; Fan, X.; Singh, D. J.; Chen, H.; Jiang, Q.; Zheng, W. T. Controlling Phase Transition for Single-Layer $\mathrm{MTe}_{2}(\mathrm{M}=\mathrm{Mo}$ and $\mathrm{W})$ : Modulation of the Potential Barrier under Strain. Phys. Chem. Chem. Phys. 2016, 18 (5), 4086-4094.

(4) Shim, J.; Oh, S.; Kang, D. H.; Jo, S. H.; Ali, M. H.; Choi, W. Y.; Heo, K.; Jeon, J.; Lee, S.; Kim, M.; Song, Y. J.; Park, J-H. Phosphorene/Rhenium Disulfide HeterojunctionBased Negative Differential Resistance Device for Multi-Valued Logic. Nat. Commun. 2016, 7, 13413. 